\title{
关于提高抄平起拨道捣固车在铁路新线曲线上 拨道作业质量的研究
}

\author{
Study on Improving the Quality of Track Lining Operation of Leveling-Lifting-Lining-Tamping \\ Machine on the Curves of New Railway Lines
}

\author{
陈金金 \\ Xin Chen
}

中铁一局集团新运工程有限公司, 中国·陕西 咸阳 712000

Xinyun Engineering Co.Ltd.ofChina Railway First Group Co.Ltd., Xianyang, Shaanxi, 712000, China

\begin{abstract}
【摘要】在大型养路机械整道过程中, 曲线正矢尤其是缓和曲线正矢超限问题较多, 达不到验标要求。论文对如何进一步发挥大型养路机械效 能, 提高曲线正矢的作业质量, 做了一些有益的探索。

【Abstract】In the process of repairing the road by large track maintenance machinery, there are many problems in curve verses, especially the over vector of positive curves, which fail to meet the requirements of inspection standard. The paper has made some useful explorations on how to give full play to the efficiency of large maintenance machinery, and improve the quality of the curve versine.
\end{abstract}

【关键词】抄平起拨道捣固车; 质量; 快速计算

Keywords \leveling-lifting-lining-tamping machine; quality; fast calculation

【DOI】http://dx.doi.org/10.26549/gcjsygl.v2i4.757

\section{1 引言}

论文针对抄平起拨道捣固车在铁路新线作业后曲线正矢 合格率普遍偏低的情况，根据铁路新线特点与抄平起拨道捣 固车作业原理, 引入既有铁路曲线养护作业方法一一绳正法, 研究了曲线拨道量采用绳正法的计算方法与绳正法在铁路新 线配合抄平起拨道捣固车作业的适用条件，大幅度提高了抄 平起拨道捣固车在铁路新线作业后曲线正矢质量。

\section{2 新线铁路大型养路机械曲线整道现状} 和特点

新线铁路开通前均要进行大机整道, 整道时按中桩提供 拨道资料,其整道作业大致有如下两种情况。

\section{1 荒道作业}

线路从上第一遍道砟荒道情况时至开通前进行多遍作 业, 每遍都要按中桩提供拨道资料。虽然进行多遍作业,但其 曲线正矢合格率均不高, 在 $70 \%$ 左右, 达不到验标要求。

\section{2 在人工初步整道后进行大型养路机械作业}

线路在人工整道后一般预留 50 80mm 的起道量, 开通前 再进行两遍大机整道。由于一般新线区段均比较长, 由于人工 拨道经验不足与工期要求紧等诸多因素影响, 线路往往拨不 到位, 特别是曲线拨道质量更差, 大型养路机械整前线路拨道
量一般都很大, $50 \mathrm{~mm}$ 以上的拨道量很多, 机械两次拨道难以 到位, 因此造成机械整道后曲线正矢较差。这种线路曲线正矢 合格率一般在 $60 \%$ 左右, 较前一种作业方式差 ${ }^{[1]}$ 。

\section{3 拨道作业误差分析}

\section{1 起拨道捣固车采用近似法拨道}

由于起拨道捣固车拨道作业原理是近似法拨道, 其本身 存在系统误差。其三点法拨道后方向残留偏差是拨道量的 1 / 3.04 , 四点法为 $1 / 6.12$ 。因此不论是三点法检测拨道还是四点 法检测拨道, 只能达到线路方向的基本圆顺, 不能完全消除线 路方向偏差, 拨道后线路仍有一定的方向偏差残留。

\section{2 提供拨道资料的拨道量大,拨道点间距大}

\section{2 .1 拨道点间距大}

由于是按线路中桩点来提供拨道点位置, 曲线拨道点距 离一般为: 缓和曲线内是 $10 \mathrm{~m}$, 圆曲线内为 $20 \mathrm{~m}$, 但精确拨道 所要求的距离为 $2.5 \mathrm{~m}$, 故拨道点的间距无法满足精确拨道间 距的要求。

\section{2 .2 拨道量比较大}

由于是新线铁路, 线路状况较差, 拨道量一般比较大, $50 \mathrm{~mm}$ 以上的拨道量大量存在, 不能符合近似法一次最大拨道 量的要求。而在 $650 \mathrm{~m}$ 以上的大半径曲线中, 三点法要求的一 
次最大拨道量仅为 $18 \mathrm{~mm}$, 四点法也只为 $36 \mathrm{~mm}$ 。

\section{3 作业对位偏差大}

捣固车作业时, GVA 对曲线桩位置输入的容许偏差为 $0.5 \mathrm{~m}$, 作业对位偏差超过这个距离, 对拨道作业质量就会有影 响。

\section{4 捣固车拨道作业装置长度有限}

捣固车作业时只能在这个测量弦线有限长度范围内对线 路进行一次性拨道作业, 不能对拨道点的位置偏差在整个曲 线范围内进行综合调整后再进行拨道作业。

\section{5 线路上钢轨在拨道作业后有回弹量}

捣固车拨道作业后钢轨存在一定程度的回弹, 特别是在 拨道量大且缺砟地段钢轨回弹量比较大，对拨道作业效果影 响严重。

由于以上因素的影响,捣固车拨道作业只能改善线路几何 形状,无法消除线路的正矢误差,曲线圆顺了, 满足了系统拨道 要求, 不论大型养路机械拨多少遍, 都很难达到满意的效果 [2]。

\section{4 绳正法在铁路新线上的应用}

绳正法是以弦线测量正矢检查曲线的圆度来调整正矢的 方法。既有铁路曲线养护采用绳正法较为普遍, 且效果较好。 我们利用其原理来研究在机械整道时提高抄平起拨道捣固车 在铁路新线曲线拨道作业时的质量措施。

\section{1 绳正法基本原理}

在计算拨道量时, 采用的计算方法较多, 但其基本原理都 是相同的。

4.1 .1 两条假定

(1)假定曲线两端切线方向不变,即曲线始终点拨量为零。 切线方向不变, 也就是曲线的转角不变。即 $\sum \mathrm{f}$ 现 $=\sum \mathrm{f}$ 计

式中: $\sum \mathrm{f}$ 现一一现场正矢总和

$\sum \mathrm{f}$ 计一一计划正矢总和

同时还要保证曲线两端直线不发生平行 移动, 即始终点拨量为零。

(2)曲线上某一点拨道时，其相邻测点在 长度上并不随之移动, 拨动后钢轨总长不变。

4.1 .2 四个基本原理

(1)等长弦分圆曲线为若干弧段，则每弧 段正矢相等,即等圆等弧的弦心距相等。

(2)曲线上任一点拨动，对相邻点均有影 响, 对相邻点正矢的影响量为拨点处拨动量 的二分之一,其方向相反。这是由于线路上钢 轨是连续的, 拨动曲线时, 某一点正矢增加, 前后两点正矢则各减少拨动量的二分之一
值; 反之, 某一点正矢拨动量减少, 前后两点正矢则随之增加 拨量的二分之一值。

(3)拨道前与拨道后整个曲线正矢总和不变。

(4)在拨道时整个曲线各测点正矢发生的增减量总和必等 于零。

基于以上假设与基本原理，在铁路新线上利用现场正矢 来计算曲线拨道量时，线路上每一点位置的拨道量都会对整 条曲线的位置产生影响，拨道后整条曲线位置相对于原线路 设计中桩位置重新进行了调整, 并确保了每一拨道点的拨后 曲线正矢都符合曲线的计划正矢要求, 因此将绳正法引入铁 路新线机械整道作业是可行的，可以大幅提高拨后曲线正矢 质量。

\section{2 拨道量的计算方法}

在计算拨道量时,采用的计算方法较多,下面我们采用两 个较为易学易算的方法进行计算举例。

4.2 .1 流水法(列表法)

表格栏目说明及计算方法:

第 1 栏, 测点: 即曲线正矢点号, 测量正矢的桩号。

第 2 栏,现场正矢: 从线路实测出来的正矢。

第 3 栏, 计划正矢: 按曲线半径和缓和曲线长计算出来的 正矢。

第 4 栏,正矢差:现场正矢与计划正矢之差。如现场正矢 大于计划正矢,其差为正, 反之为负。

第 5 栏,差的累计:正矢差的代数和。测点差的累计与后 点正矢差之和即为后点的差的累计,称为“斜加平写”,如表中 第 $4 、 5$ 栏箭头所示。

第 6 栏,半拨量:即正矢差累计的合计,拨距的一半。前点 半拨量加前点差的累计等于该点的半拨量, 称为“平加下写”,

如表 1 中第 $5 、 6$ 栏箭头所示。

表 1 流水法计算表

\begin{tabular}{|c|c|c|c|c|c|c|c|c|}
\hline 测点 & 现场正矢 & 计划正矢 & 正矢差 & 差的累计 & 半拨量 & 全拨量 & 校核 & 备注 \\
\hline 1 & 2 & 3 & 4 & 5 & 6 & 7 & 8 & 9 \\
\hline 1 & 2 & 2 & 0 & $0 \leqslant$ & 0 & 0 & $0+0+2=2$ & $\mathrm{ZH}$ \\
\hline 2 & 12 & 10 & +2 & +2 & $\triangle 0$ & 0 & $0+0-2+12=10$ & \\
\hline 3 & 17 & 20 & $-3 \longleftarrow$ & -1 & $\geq+2$ & +4 & $0+(+4)-1+17=20$ & \\
\hline 4 & 26 & 30 & $-4 \longleftarrow$ & -5 & $\mathbf{- 1}$ & +2 & $-2+(+2)+4+26=30$ & \\
\hline 5 & 42 & 40 & +2 & -3 & -4 & -8 & $-17(-8)+7+42=40$ & \\
\hline 6 & 51 & 48 & +3 & 0 & -7 & -14 & $+4+(-14)+7+51=48$ & HY \\
\hline 7 & 56 & 50 & +6 & +6 & -7 & -14 & $+7+(-14)+1+56=50$ & \\
\hline 8 & 46 & 50 & -4 & +2 & -1 & -2 & $+7+(-2)-1+46=50$ & \\
\hline 9 & 44 & 50 & -6 & -4 & +1 & +2 & $+1+(+2)+3+44=50$ & \\
\hline 10 & 53 & 50 & +3 & -1 & -3 & -6 & $-1+(-6)+4+53=50$ & \\
\hline 11 & 49 & 48 & +1 & 0 & -4 & -8 & $+3+(-8)+4+49=48$ & $\mathrm{YH}$ \\
\hline 12 & 43 & 40 & +3 & +3 & -4 & -8 & $+4+(-8)+1+43=40$ & \\
\hline 13 & 27 & 30 & -3 & 0 & -1 & -2 & $+4+(-2)+1+27=30$ & \\
\hline 14 & 21 & 20 & +1 & +1 & -1 & -2 & $+1+(-2)+0+21=20$ & \\
\hline 15 & 9 & 10 & -1 & 0 & 0 & 0 & $+1+0+0+9=10$ & \\
\hline 16 & 2 & 2 & 0 & 0 & 0 & 0 & $0+0+0+2=2$ & $\mathrm{HZ}$ \\
\hline 17 & 0 & 0 & 0 & 0 & 0 & 0 & 0 & \\
\hline
\end{tabular}


第 7 栏, 全拨量: 测点的拨道量,为半拨量的 2 倍。

第 8 栏,校核: 校核计算的正误。根据拨动一点,相邻测点 各动半数的原理, 核对各点拨后正矢是否与计划正矢相等, 如 不等,则计算有误,应重新计算。

将测点全拨量的一半符号相反地分给前后点,如第 4 点 全拨量 +2 , 分给前后点各 -1 。测点全拨量、影响量与现场正矢 相加的代数和即为拨后正矢, 如第 9 点, 将第 8 点的前影响 量 +1 , 加拨道量 +2 , 加第 10 点后影响量 +3 , 再加现场正矢 44 , 即可得拨后正矢 $50^{[3]}$ 。

\subsection{2 简易法的应用}

计算说明:

(1)调整量: 如个别点现场正矢与计划正矢误差较大, 为减 小拨道量和计算方便, 可预先个别调整。按拨动一点, 其前后 各影响一半且符号相反的原则进行调整。如测点 9 的现场正 矢 71 可以调整 -8 , 其前后点现场正矢各调整 +4 。

(2)正矢差: 计划正矢减去现场正矢之差。这与流水法计算 方法不同。

(3)拨道量计算: 测点正矢差与前点拨道量的前影响量之 代数和为该点的拨道量, 如测点 7 的正矢差为 -4 , 与测点 6 拨 道量的前影响量 +1 相加即可得出拨道量 -3 ; 同理, 该点拨道 量的后影响量与后点正矢差代数和就是后点的拨道量。拨道 量的前后影响量为其一半,符号不变,在计算过程中 0.5 可不
计, 如测点 7 的拨道量是 -3 , 其前后影响量则各为 -1 。

(4)如经第一次计算后, 后影响量不为零, 则须进行第二次 计算。第一次计算的后影响量与第二次的前影响量的代数和, 即为第二次的拨道量。以后的计算同 3 。

(5)按照上述方法, 进行一次、二次甚至多次计算, 直至后 影响量全部为零。

(6)将几次计算的拨道量加起来, 就可得出总拨道量。如在 最后一次计算后, 后影响量不等于零而等于 1 , 则可不再计算, 因为此时测点前后影响量都为 0.5 , 在计算过程中是不计 0.5 的。但在计算总拨道量时,除了将调整量和前几次计算的拨道 量相加外,还应加上最后一次计算的后影响量。

(7)校核。根据拨动一点, 相邻测点各动半数的原理, 核对 测点的拨后正矢与计划正矢是否相符。拨后正矢的计算方法 与流水法相同。拨后正矢计算完成后与计划正矢进行对照检 查。如误差在 $2 \mathrm{~mm}$ 以内, 则计算无误(误差是由取舍 0.5 造成 的), 反之则计算有误。

4.2.3 新线铁路在大机整道时采用绳正法应注意的事项

(1)作业前线路状况应比较好。线路经过人工或大机初整 道, 曲线较圆顺, 线路已基本按中桩拨到位, 特别是曲线始终 点要必须到位。

(2)需要特别注意的是,新线铁路与既有线不同,计算时曲 线计划正矢不能调整。大机拨后曲线正矢检查是按设计计划

简易法计算表

\begin{tabular}{|c|c|c|c|c|c|c|c|c|c|c|c|c|c|c|c|c|c|c|c|}
\hline \multirow[b]{2}{*}{ 测点 } & \multirow[b]{2}{*}{$\begin{array}{l}\text { 计划 } \\
\text { 正矢 }\end{array}$} & \multirow[b]{2}{*}{$\begin{array}{l}\text { 现场 } \\
\text { 正矢 }\end{array}$} & \multicolumn{3}{|c|}{ 调整量 } & \multirow{2}{*}{$\begin{array}{c}\text { 调整 } \\
\text { 后正 } \\
\text { 矢 }\end{array}$} & \multirow[b]{2}{*}{$\begin{array}{c}\text { 正矢 } \\
\text { 差 }\end{array}$} & \multicolumn{2}{|c|}{ 第一次计算 } & \multicolumn{4}{|c|}{ 第二次计算 } & \multicolumn{3}{|c|}{ 第三次计算 } & \multirow[b]{2}{*}{$\begin{array}{l}\text { 总拨 } \\
\text { 道量 }\end{array}$} & \multirow[b]{2}{*}{ 校核 } & \multirow[b]{2}{*}{ 备注 } \\
\hline & & & $\begin{array}{l}\text { 前影 } \\
\text { 响量 }\end{array}$ & $\begin{array}{c}\text { 拨道 } \\
\text { 量 }\end{array}$ & $\begin{array}{l}\text { 后影 } \\
\text { 响量 }\end{array}$ & & & $\begin{array}{l}\text { 前影 } \\
\text { 响量 }\end{array}$ & $\begin{array}{c}\text { 拨道 } \\
\text { 量 }\end{array}$ & $\begin{array}{l}\text { 后影 } \\
\text { 响量 }\end{array}$ & $\begin{array}{l}\text { 前影 } \\
\text { 响量 }\end{array}$ & $\begin{array}{c}\text { 拨道 } \\
\text { 量 }\end{array}$ & $\begin{array}{l}\text { 后影 } \\
\text { 响量 }\end{array}$ & $\begin{array}{l}\text { 前影 } \\
\text { 响量 }\end{array}$ & $\begin{array}{c}\text { 拨道 } \\
\text { 量 }\end{array}$ & $\begin{array}{l}\text { 后影 } \\
\text { 响量 }\end{array}$ & & & \\
\hline 0 & 0 & 0 & & & & 0 & 0 & 0 & 0 & 0 & 0 & 0 & 0 & 0 & 0 & 0 & 0 & 0 & \\
\hline 1 & 3 & 3 & & & & 3 & 0 & $0-$ & $0^{-}$ & 0 & 0 & $0^{-}$ & $0-$ & $0-$ & $=0^{-}$ & 0 & 0 & 3 & $\mathrm{ZH}$ \\
\hline 2 & 16 & 16 & & & & 16 & 0 & 0 & 0 & +1 & $0 \longleftarrow$ & +1 & -1 & 0 & -1 & 0 & 0 & 16 & \\
\hline 3 & 31 & 35 & & & & 35 & -4 & 0 & +2 & -3 & 0 & -3 & 0 & 0 & 0 & 0 & -1 & 31 & \\
\hline 4 & 47 & 54 & & & & 54 & -7 & +1 & -6 & 0 & -1 & -1 & 0 & 0 & 0 & 0 & -7 & 47 & \\
\hline 5 & 60 & 58 & & & & 58 & +2 & -3 & -1 & +1 & 0 & +1 & 0 & 0 & 0 & 0 & 0 & 60 & HY \\
\hline 6 & 63 & 60 & & & & 60 & +3 & 0 & +3 & -1 & 0 & -1 & 0 & 0 & 0 & 0 & +2 & 63 & \\
\hline 7 & 63 & 67 & & & & 67 & -4 & +1 & -3 & 0 & 0 & 0 & 0 & 0 & 0 & 0 & -3 & 63 & \\
\hline 8 & 63 & 58 & & & +4 & 62 & +1 & -1 & 0 & 0 & 0 & 0 & 0 & 0 & 0 & 0 & 0 & 63 & \\
\hline 9 & 63 & 71 & & -8 & & 63 & 0 & 0 & 0 & 0 & 0 & 0 & 0 & 0 & 0 & 0 & -8 & 63 & \\
\hline 10 & 63 & 60 & +4 & & & 64 & -1 & 0 & -1 & 0 & 0 & 0 & 0 & 0 & 0 & 0 & -1 & 63 & \\
\hline 11 & 63 & 63 & & & & 63 & 0 & 0 & 0 & -1 & 0 & -1 & 0 & 0 & 0 & 0 & -1 & 62 & \\
\hline 12 & 63 & 67 & & & & 67 & -4 & 0 & -2 & +1 & 0 & +1 & 0 & 0 & 0 & 0 & -1 & 63 & \\
\hline 13 & 63 & 60 & & & & 60 & +3 & -1 & +2 & 0 & 0 & 0 & 0 & 0 & 0 & 0 & +2 & 62 & \\
\hline 14 & 63 & 69 & & & -5 & 64 & -1 & +1 & 0 & 0 & 0 & 0 & 0 & 0 & 0 & 0 & 0 & 63 & \\
\hline 15 & 63 & 52 & & +10 & & 62 & +1 & 0 & +1 & 0 & 0 & 0 & 0 & 0 & 0 & 0 & +11 & 62 & \\
\hline 16 & 63 & 67 & -5 & & & 62 & +1 & 0 & +1 & +1 & 0 & +1 & +1 & 0 & +1 & 0 & +3 & 62 & \\
\hline 17 & 63 & 60 & & & & 60 & +3 & 0 & +3 & +2 & 0 & +2 & +1 & 0 & +1 & 0 & +6 & 62 & \\
\hline 18 & 63 & 59 & & & & 59 & +4 & +1 & +5 & +1 & +1 & +2 & 0 & 0 & 0 & 0 & +7 & 61 & \\
\hline 19 & 63 & 62 & & & & 62 & +1 & +2 & +3 & 0 & +1 & +1 & 0 & 0 & 0 & 0 & +4 & 63 & \\
\hline 20 & 63 & 64 & & & & 64 & -1 & +1 & 0 & 0 & 0 & 0 & 0 & 0 & 0 & 0 & 0 & 62 & \\
\hline 21 & 60 & 59 & & & & 59 & +1 & 0 & +1 & -1 & 0 & -1 & -1 & 0 & -1 & 0 & -1 & 60 & $\mathrm{YH}$ \\
\hline 22 & 47 & 49 & & & & 49 & -2 & 0 & -2 & -2 & 0 & -2 & -1 & 0 & -1 & 0 & -5 & 47 & \\
\hline 23 & 31 & 37 & & & & 37 & -6 & -1 & -5 & -1 & -1 & -2 & 0 & 0 & 0 & 0 & -7 & 31 & \\
\hline 24 & 16 & 16 & & & & 16 & 0 & -2 & -2 & 0 & -1 & -1 & 0 & 0 & 0 & 0 & -3 & 16 & \\
\hline 25 & 3 & 3 & & & & 3 & 0 & -1 & -1 & 0 & 0 & 0 & 0 & 0 & 0 & 0 & -1 & 3 & $\mathrm{HZ}$ \\
\hline 26 & 0 & 0 & & & & 0 & 0 & 0 & 0 & 0 & 0 & 0 & 0 & 0 & 0 & 0 & 0 & 0 & \\
\hline
\end{tabular}


正矢进行的, 调整计划正矢虽然减小了拨道量, 但会大幅降低 拨后正矢合格率。为减小拨道量, 可以对正矢偏差较大的现场 正矢预先个别调整。

不调整计划正矢可能会使曲线两端产生较小的拨道量, 故曲线头尾与直线的顺接要用激光矫直系统来完成。

(3)如在曲线分桩时考虑大机作业特点, 把不足 $10 \mathrm{~m}$ 长的 破链划分在圆曲线上, 使曲线两端桩点落在 “ZH” 和 “ HZ” 点 上, 利用激光矫直系统, 则会基本消除曲线两端 “鸡头” 的产 生, 提高曲线的圆顺度和正矢质量。

(4)在捣固车进行曲线作业时线路上道砟要饱满, 一定要 起、拨、捣作业一起进行, 如仅进行拨道作业, 钢轨的回弹量大 会使拨后效果不明显。

(5)由于测量曲线正矢的弦线长为 $20 \mathrm{~m}$, 我们通常每 $10 \mathrm{~m}$ 测量一次曲线现场正矢, 也就是每 $10 \mathrm{~m}$ 我们可以计算提供一 处拨道量。但为加密拨道间距, 我们在现场混凝十枕面上标注 拨道量时, 可以在前后拨道点中间位置加设拨道点, 将拨道点 加密成 $5 \mathrm{~m}$, 并根据现场综合考虑中间点拨道量。加密拨道点 后捣固车拨道作业质量会更好。

(6)给前司机室操作人员提供拨道量表, 操作司机在往 GVA 输入拨道量时会根据前一点拨道量与线路现状及时调整

\section{（上接第 90 页）}

合作和并购的机会, 不断扩大自身的规模, 完成原始积累, 为 进一步的扩张做准备。

\section{3 房地产项目管理过程中的对策}

\subsection{1 建立多级架构和高效流程管理机制}

对于管理链条过长的问题, 可以探索建立多层机构来管 理, 例如建立集团公司-区域公司-城市公司-片区公司-项目 公司的多层级架构,通过统一的信息化系统, 集团制定各种政 策和流程, 所有层级的公司都要遵守和执行。这样, 可以保证 信息在各层级之间传播的无障碍性, 以及在流程设计上, 项目 公司的行动需要各层级领导审批，保证了集团政策落地的高 效性。

\subsection{2 标准化流程推广, 人人问责}

对于提高快周转, 对各个部门的要求很高, 对于这个问 题, 可以通过标准化流程控制来推进,即在集团层面创设不同 的业务场景, 对于不同场景提出统一的安排和要求, 建立标准 模板; 项目公司在自身项目特点的基础上, 可以进行改进, 报 集团批准后推广。同时, 各个业务部门对于每个项目都确定特 定的对接人, 做到每件事情都有人负责, 无法推脱 ${ }^{[4]}$ 。

\section{3 .3 发现自身的核心竞争}

对于行业竞争的问题, 需要房地产公司发现自身的核心
前后拨道点问线路每一处拨道量，从而使捣固车拨道作业质 量能更进一步提高。

\section{5 新线铁路大机整道应用绳正法后的效果}

大机整道后曲线正矢质量提高显著。根据中南部通道铁 路、太中银铁路、甘泉铁路等十多条新线铁路施工经验及采用 改进措施的实践,引入绳正法配合抄平起拨道捣固车作业后, 曲线正矢质量大幅提高, 正矢合格率显著上升, 特别是缓和曲 线正矢合格率提升明显，曲线拨后正矢平均合格率由原来的 不足 60\%跃升到平均 $90 \%$ 以上, 很多曲线正矢合格率达到了 $100 \%$, 质量上了一个大台阶。实践证明在铁路新线机械整道 时, 采用绳正法配合作业是一个能大幅提高曲线正矢质量的 较好途径, 效果显著, 绳正法也由此在本单位迅速得以大范围 推广并产生了良好的经济与社会效益。

\section{参考文献:}

[1]吴桂清.铁路搊固车设备状态与作业质量在线监测方法及应用 [D].长沙:湖南大学, 2012 .

[2]杨锴. 浅议大型捣固车在铁路曲线维修中的技术措施[J. 铁道建 设,2011(2):7-9.

[3]陆亦群, 高春雷, 王发灯. 提高既有捣固车作业精度的研究 [J].中 国铁路,2006(12):45-47.

竞争力, 以此为基础不断外延,比如与政府关系良好的公司, 拿地能力强; 对于快周转强的公司, 加强快周转的能力。在不 断加强自身核心竞争力的同时,积极寻求合作,与其他房地产 公司强强联合, 共进退, 共发展 ${ }^{[\wp]}$ 。

\section{4 结语}

从上面的分析可以看出, 房地产行业的项目管理过程中 确实存在很多问题, 而且大部分都是房地产行业的特点所决 定的, 很难从根本上解决, 所以, 如何对管理进行变革和改变, 如何有效解决存在的问题, 是房地产公司需要考虑的主要方 向。

\section{参考文献:}

[1]刘玉胜.房地产开发项目质量管理浅析 [J].知识经济,2013(19): $118+130$.

[2]石庆省.房地产建筑施工技术管理及控制研究[J].住宅与房地 产, 2015(28):83.

[3]左斌. 房地产开发建设的技术管理[J].长春工程学院学报(社会 科学版),2001(03):32-33+56.

[4]张军明.浅析房地产开发工程的成本控制及管理措施[J].科技 创新与应用,2014(15):258.

[5]王敏.分析房地产开发项目造价控制与成本管理 [J].住宅与房 地产,2015(28):150. 